\title{
Squeezing the periodicity of Néel-type magnetic modulations by enhanced Dzyaloshinskii-Moriya interaction of $4 d$ electrons
}

\author{
Ádám Butykai $\mathbb{D}^{1,2 凶}{ }^{1}$, Korbinian Geirhos $\mathbb{D}^{3}$, Dávid Szaller ${ }^{1,4}$, László F. Kiss $\mathbb{D}^{5}$, László Balogh ${ }^{1}$, Maria Azhar $\mathbb{C}^{6}$, Markus Garst ${ }^{6,7}$, \\ Lisa DeBeer-Schmitt $\mathbb{1}^{8}$, Takeshi Waki ${ }^{9}$, Yoshikazu Tabata ${ }^{9}$, Hiroyuki Nakamura $\mathbb{1}^{9}$, István Kézsmárki ${ }^{3}$ and Sándor Bordács $\mathbb{1}^{1,10 凶}$
}

In polar magnets, such as $\mathrm{GaV}_{4} \mathrm{~S}_{8}, \mathrm{GaV}_{4} \mathrm{Se}_{8}$ and $\mathrm{VOSe}_{2} \mathrm{O}_{5}$, modulated magnetic phases namely the cycloidal and the Néel-type skyrmion lattice states were identified over extended temperature ranges, even down to zero Kelvin. Our combined small-angle neutron scattering and magnetization study shows the robustness of the Néel-type magnetic modulations also against magnetic fields up to $2 \mathrm{~T}$ in the polar $\mathrm{GaMo}_{4} \mathrm{~S}_{8}$. In addition to the large upper critical field, enhanced spin-orbit coupling stabilize cycloidal, Néel skyrmion lattice phases with sub-10 $\mathrm{nm}$ periodicity and a peculiar distribution of the magnetic modulation vectors. Moreover, we detected an additional single-q state not observed in any other polar magnets. Thus, our work demonstrates that noncentrosymmetric magnets with $4 d$ and $5 d$ electron systems may give rise to various highly compressed modulated states.

npj Quantum Materials (2022)7:26; https://doi.org/10.1038/s41535-022-00432-y

\section{INTRODUCTION}

In the presence of strong spin-orbit coupling (SOC) topologically non-trivial states of condensed matter emerge such as the surface states of topological insulators ${ }^{1,2}$, Dirac and Weyl fermions $s^{3,4}$ or Majorana particles ${ }^{5,6}$. In spin systems, the first order manifestation of the SOC is the antisymmetric Dzyaloshinskii-Moriya interaction (DMI), which gives rise to spin spirals and skyrmion lattice (SkL) states in non-centrosymmetric compounds ${ }^{7-9}$. The non-trivial topology of skyrmions ${ }^{10,11}$, as well as their interaction with conduction electrons ${ }^{12}$, electric fields ${ }^{13-16}$ and spin-waves ${ }^{17-19}$ motivated intense research exploring possible applications in next-generation data storage and microwavefrequency spintronic devices ${ }^{20-23}$.

The SkL phase was first observed in the chiral cubic helimagnet $\mathrm{MnSi}$ with moderate $\mathrm{SOC}^{24}$. In cubic helimagnets the symmetrydictated form of DMI enables Bloch-type magnetic modulations, i.e., spin helices and Bloch-skyrmions. This Bloch-type SkL, comprising $q$-vectors perpendicular to the direction of the applied field, is stabilized by thermal fluctuations over the energetically favoured longitudinal conical state, only in the close vicinity of the Curie temperature ${ }^{24,25}$. Cubic magnetocrystalline anisotropies that are higher-order terms in the SOC determine the orientation of helical order at zero field and induce small deflections of the SkL planes for fields applied along low-symmetry crystallographic directions ${ }^{25,26}$. The increasing strength of the SOC was studied in $\mathrm{Mn}\left(\mathrm{Si}_{1-x} \mathrm{Ge}_{x}\right)$ by replacing $\mathrm{Si}$ with heavier $\mathrm{Ge}^{27}$. As a result, the periodicity of the magnetic modulation decreases and the SkL state is transformed to a hedgehog-lattice state. The enhanced cubic anisotropy can also lead to SkL states at low temperatures, without relying on stabilization by thermal fluctuations ${ }^{28,29}$.

For potential memory and spintronic applications a further reduction in the skyrmion size is desired, which can be achieved through the enhancement of the SOC. In the vast majority of the skyrmion host crystals reported to date, the magnetism is governed by the $3 d$ electrons of transition metals, such as $\mathrm{V}, \mathrm{Mn}$, $\mathrm{Fe}, \mathrm{Co}, \mathrm{Cu}$. Here, we demonstrate the emergence of particularly robust modulated magnetic phases in the $4 d$ polar magnet, $\mathrm{GaMo}_{4} \mathrm{~S}_{8}{ }^{30-34}$. In the rhombohedral phase, our small-angle neutron scattering (SANS) data (Fig. 1c) show that the strong SOC reduces the periodicity of the magnetic structure to $\lambda \approx$ $9.8 \mathrm{~nm}$, being one of the shortest modulation observed in bulk crystals hosting the SkL state due to DMI. Moreover, it modifies the distribution of the $q$-vectors, as illustrated in Fig. $1 \mathrm{~d}$, which we explain by an effective Landau theory containing a cubic anisotropy term in addition to the axial anisotropy, inherent to the rhombohedral state. Our temperature, magnetic field and angular dependent magnetization and electric polarization measurements reveal a complex phase diagram where we attributed phases to the cycloidal and SkL states and observed a third modulated magnetic phase. Moreover, we found that the modulated spin states extend up to fields as high as $2 \mathrm{~T}$, which further support their robustness.

Spin cycloids and Néel SkL have been found recently in the polar phase of the lacunar spinels $\mathrm{GaV}_{4} \mathrm{~S}_{8}$ and $\mathrm{GaV}_{4} \mathrm{Se}_{8}{ }^{35-38}$, which are narrow-gap multiferroic semiconductors ${ }^{39,40}$. The polar rhombohedral structure (space group $R 3 \mathrm{~m}$ ) develops via a cooperative JahnTeller distortion driven by the unpaired electron of each $\mathrm{V}_{4}$ cluster with spin $S=1 / 2$ occupying a triply degenerate orbital in the hightemperature cubic state $(F \overline{4} 3 m)^{39}$. The rhombohedral distortion can occur as elongation of the unit cell along any of the four $<111>$-type cubic directions, thus, four rhombohedral domain states, that we denote by $\mathrm{P}_{1-4}$, are present below the structural transition at $\sim 42 \mathrm{~K}^{37,39,41}$. (Throughout this paper we use the pseudo-cubic notation.) In contrast to the chiral cubic helimagnets, in these lacunar spinels the cycloidal character of the modulations with $q$-vectors restricted to the plane perpendicular to the rhombohedral axis (Fig. 1a, b) enhances the stability range of the SkL phase $35,37,42$. In both compounds, SANS experiments revealed

\footnotetext{
${ }^{1}$ Department of Physics, Budapest University of Technology and Economics, 1111 Budapest, Hungary. ${ }^{2}$ Condensed Matter Research Group of the Hungarian Academy of Sciences, 1111 Budapest, Hungary. ${ }^{3}$ Experimental Physics V, Center for Electronic Correlations and Magnetism, University of Augsburg, 86135 Augsburg, Germany. ${ }^{4}$ Institute of Solid State Physics, TU Wien, 1040 Vienna, Austria. ${ }^{5}$ Department of Experimental Solid State Physics, Institute for Solid State Physics and Optics, Wigner Research Centre for Physics, 1121 Budapest, Hungary. ${ }^{6}$ Institut für Theoretische Festkörperphysik, Karlsruhe Institute of Technology, 76131 Karlsruhe, Germany. ${ }^{7}$ Institute for Quantum Materials and Technology, Karlsruhe Institute of Technology, 76131 Karlsruhe, Germany. ${ }^{8}$ Neutron Scattering Division, ORNL, Oak Ridge, TN 37831, USA. ${ }^{9}$ Department of Materials Science and Engineering, Kyoto University, Kyoto 606-

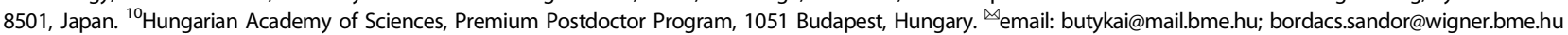





Fig. 1 A comparison of the reciprocal-space structure of the modulation wavevectors in $\mathrm{GaV}_{4} \mathrm{~S}_{8}$ and $\mathrm{GaMo}_{4} \mathrm{~S}_{8}$. a The tomographic SANS image measured at $12 \mathrm{~K}$ in $\mathrm{GaV}_{4} \mathrm{~S}_{8}$ and $\mathbf{b}$ its graphical representation. The scattering pattern contains four rings of $q$ vectors represented by distinct colours in (b), each corresponding to one of the rhombohedral domain states. $c$ The distribution of the magnetic wavevectors observed at $2 \mathrm{~K}$ in $\mathrm{GaMo}_{4} \mathrm{~S}_{8}$ and $d$ its schematic view. The rings are deflected from the $\{111\}$-type planes in the segments between the $\langle 110\rangle$ directions in an alternating manner.

magnetic modulations with wavelengths of $\lambda \sim 20 \mathrm{~nm}^{35-37,43}$, indicating a similar ratio of the exchange interactions and the DMls.

In the compound $\mathrm{GaMo}_{4} \mathrm{~S}_{8}$ studied here, the tetrahedral $\mathrm{Mo}_{4}$ clusters carry an unpaired hole with spin $S=1 / 2^{39}$. Correspondingly, the cubic state is rhombohedrally distorted by the compression of the unit cell along one of the <111>-type directions ${ }^{31}$. Although, Rastogi et al., pointed out the importance of the correlation in $\mathrm{GaMo}_{4} \mathrm{~S}_{8}$ and found a rich magnetic phase diagram below $T_{C}=19 \mathrm{~K}$ long ago ${ }^{30}$, the spin ordering patterns of these phases have not been studied.

\section{RESULTS}

Wavevector distribution of the magnetic cycloid at zero field We explored the zero-field modulated magnetic states of $\mathrm{GaMo}_{4} \mathrm{~S}_{8}$ by SANS experiments. (The scattering geometry is shown in Supplementary Fig. 4) The scattered intensity was recorded in zero-field at $2 \mathrm{~K}$ upon the $180^{\circ}$ rotation of the sample in $1^{\circ}$ steps, with an acquisition time of $120 \mathrm{~s}$ at each angle. The background signal was measured in the paramagnetic phase at $25 \mathrm{~K}$ following the same procedure. The scattering images were averaged over a $10^{\circ}$ moving window in the rotation angle to improve the signal-tonoise ratio. Figure $2 a-d$ shows the SANS images obtained on four high-symmetry planes, namely the (111), (110), (11 $\overline{2})$ and (001) planes. A pixel-wise adaptive Wiener filter, assuming Gaussian noise, was applied for better visualization.

The q-dependence of the scattering intensity in the (111) plane, averaged over the polar angles, was fitted by a Gaussian, yielding $|\mathbf{q}|=0.64 \mathrm{~nm}^{-1}$ for the length of the modulation vectors with a FWHM of $0.2 \mathrm{~nm}^{-1}$, which corresponds to a real-space periodicity of $\lambda \approx 9.8 \mathrm{~nm}$. The uncertainty of $|\mathbf{q}|$ mainly originates from the broad and anisotropic distribution of the scattering intensity. Whereas the Curie temperature is close to that of $\mathrm{GaV}_{4} \mathrm{Se}_{8}$, suggesting a similar strength of the symmetric exchange $(J)$ in the two compounds, the modulation wavelength in $\mathrm{GaMo}_{4} \mathrm{~S}_{8}$ is roughly half of that in $\mathrm{GaV}_{4} \mathrm{~S}_{8}{ }^{35}$ and $\mathrm{GaV}_{4} \mathrm{Se}_{8}{ }^{37}$, implying a stronger DMI coupling $(D)$, as $\lambda \propto J / D$.

Over the wide-angle rotation experiment, each scattering image represents a planar cross section of the three-dimensional distribution of the $q$-vectors, where the azimuthal angle of the vertical slicing plane is varied via stepwise rotation of the sample. The 3D scattering pattern was reconstructed using the whole set of the cross section images. In order to enhance the signal-tonoise ratio and to eliminate the asymmetries of the scattering pattern introduced by imbalances between the populations of the different structural domain states, the 3D scattering pattern was symmetrized for all the symmetry operations of the cubic $T_{d}$ point group (for more details, see Supplementary Notes 2, 3 and Supplementary Figs. 5, 6). Figure 2e-h display the symmetrized image as viewed from the different high-symmetry directions.

It is instructive to compare the reciprocal-space $q$-distributions in $\mathrm{GaV}_{4} \mathrm{~S}_{8}$ and $\mathrm{GaMo}_{4} \mathrm{~S}_{8}$, as shown in Fig. $1 \mathrm{a}$ and $\mathrm{c}$, respectively. The neutron scattering data collected in the zero-field cycloidal phase of $\mathrm{GaV}_{4} \mathrm{~S}_{8}$ at $12 \mathrm{~K}$ is reproduced from ref. ${ }^{36}$. In both compounds the cycloidal $q$-vectors are distributed over four intersecting rings corresponding to the four structural domain states. The ring structure, instead of six well-defined Bragg spots, is due to static orientational disorder of the q-vectors, as discussed in ref. ${ }^{36}$. However, in contrast to $\mathrm{GaV}_{4} \mathrm{~S}_{8}$, where the modulation vectors are evenly distributed over rings restricted to the $\{111\}$-type planes, in $\mathrm{GaMo}_{4} \mathrm{~S}_{8}$, the four rings of the $q$-vectors wave out of the $\{111\}$ planes, crossing them only along the $<110>$-type directions. This waving pattern of the $q$-vectors preserves the three-fold rotational symmetry of the rhombohedral structure as highlighted by the schematic image in Fig. 1d.

To explain the distribution of the $q$-vectors, the following effective Landau potential for the unit vector $\hat{\mathbf{q}}$ is considered with its $x, y, z$ components defined in the cubic setting,

$\mathcal{V}(\hat{\mathbf{q}})=(\hat{\mathbf{n}} \hat{\mathbf{q}})^{2}+a\left(\hat{q}_{x}^{4}+\hat{q}_{y}^{4}+\hat{q}_{z}^{4}\right)+\ldots$

The first term describes the uniaxial anisotropy emerging in the rhombohedral phase with the $\hat{\mathbf{n}}$ unit vector parallel to any of the four <111>-type polar axes, and the second term is the lowestorder term compatible with the cubic $\mathrm{T}_{d}$ symmetry. The length of the $q$-vectors is essentially fixed by the DMI, $q \sim D / J$, which is consistent with the experimental SANS data. The coefficient $a$ parameterizing the relative strengths of the two terms in Eq. (1) is thus effectively of second order in the SOC. The first term favours the confinement of the $q$-vectors normal to the polar axes, $\hat{\mathbf{n}}$, as imposed by the DMI. The waving of the $q$-vectors out of the $\{111\}$ planes is captured by the second term. On the microscopic level it represents magnetocrystalline contributions to the GinzburgLandau theory for the magnetization that are effectively of fourth order in the SOC.

The minimal-energy solutions to Eq. (1) are sought by parametrizing $\hat{\mathbf{q}}$ in spherical coordinates. The polar angle, $\Theta$ is chosen with respect to the polar axis $\hat{\mathbf{n}} \|<111\rangle$ of each domain. The azimuthal angle, $\Phi$ is enclosed between the in-plane component of $\hat{\mathbf{q}}$ and one of the corresponding $\langle 1 \overline{1} 0\rangle$ directions. In the limit of weak SOC, $a$ is negligible and the wavevectors are basically in-plane $(\Theta \approx \pi / 2)$, since the spirals are degenerate with respect to the azimuthal angle $\Phi$. This gives rise to an equal distribution on circles, as observed for $\mathrm{GaV}_{4} \mathrm{~S}_{8}$ in Fig. $1 \mathrm{a}$. Expanding the potential in $\Theta$ around $\pi / 2$ up to the second order and minimizing one obtains for the deviations from the circle

$\Theta(\Phi)=\frac{\pi}{2}+\frac{\sqrt{2}}{3} \frac{a}{1+a} \sin (3 \Phi)$ 
a


b


C



g d

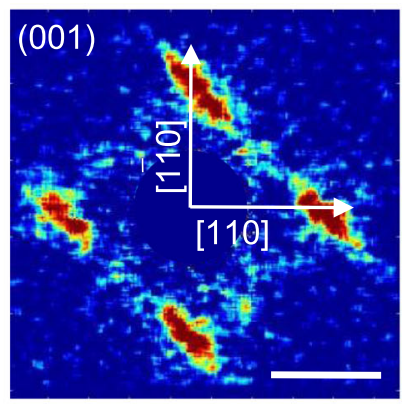

h

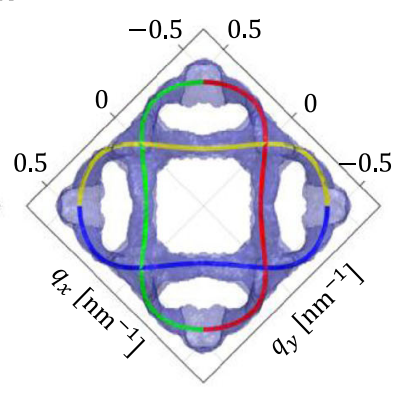

Fig. 2 SANS images of $\mathrm{GaMo}_{4} \mathrm{~S}_{8}$ and reciprocal space distribution of the cycloidal wavevectors. In panels a-d SANS images are collected at $2 \mathrm{~K}$. The scale bars represent $0.5 \mathrm{~nm}^{-1}$. In $\mathbf{e}-\mathbf{h}$, the q-vector distribution is shown from the direction of the neutron beam in (a-d), respectively. The perturbative solution of the model potential in Eq. (1) fitted to the experimental data is visualized by solid curves plotted over the experimental data. The four different colours represent scattering from the four rhombohedral domain states.

that describes the waving of $\mathbf{q}$ in $\mathrm{GaMo}_{4} \mathrm{~S}_{8}$. The least-square fitting of the data to Eq. (2) yields $a=-0.14 \pm 0.003$ and $|\mathbf{q}|=0.64 \mathrm{~nm}^{-1}$ $\pm 10^{-3}(\lambda=9.81 \mathrm{~nm})$. As shown in Fig. 2e-h, the fitted model is in excellent agreement with the SANS data, indicating the significant influence of cubic anisotropies in $\mathrm{GaMo}_{4} \mathrm{~S}_{8}$ as opposed to $\mathrm{GaV}_{4} \mathrm{~S}_{8}$ in accord with the stronger atomic SOC of Mo. We note that for $\mathrm{GaV}_{4} \mathrm{~S}_{8}$ we find $a=0 \pm 0.04$ within the experimental precision. The uncertainty is mainly determined by the image preconditioning rather than the accuracy of the fitting, for details see Supplementary Notes 2 and 3.

The tilting of $\mathbf{q}$ out of the $\{111\}$ planes as well as higher-order SOC represented by additional terms $\hat{q}_{x}^{6}+\hat{q}_{y}^{6}+\hat{q}_{z}^{6}$ in the Landau potential break the degeneracy of q-vectors around the ring and favour either $\langle 1 \overline{1} 0\rangle$ or $\langle 11 \overline{2}\rangle$-type directions. However, within our experimental accuracy, we were not able to resolve an enhanced intensity along any of these directions, see Supplementary Notes 3 and Supplementary Fig. 3.

\section{Magnetic phase diagram at finite magnetic fields}

We explore the modulated magnetic states stabilized by the interplay of external magnetic fields and the strong SOC of $\mathrm{GaMo}_{4} \mathrm{~S}_{8}$. Due to the polar symmetry of this compound, the phase diagram may depend on the angle between the polar axis and the field, thus, we measured longitudinal magnetic and differential magnetoelectric susceptibility at $10 \mathrm{~K}$ while the field was rotated in finite steps within the (110) plane between successive field sweeps. The obtained data are respectively shown in Fig. 3a, b as colour maps over the field magnitude-orientation plane, where the angle $\phi$ was measured from the [111] axis as sketched in Fig. 3c. The critical fields identified as peaks or sharp steps in the susceptibility and magnetocurrent curves are indicated by lines in Fig. 3a, b. (Field scans measured at a few high symmetry directions are shown in Supplementary Figs. 2 and 3.)

Compared to the early magnetization study performed on powder samples ${ }^{30}$, in the single crystal sample, we resolved more than two phase transitions strongly depending on the direction of the field. The interpretation of the complicated angulardependent pattern of the anomalies can be simplified by assuming that all four polar domain states are present in the sample. Anomalies and their replica appearing at positions shifted by $\sim 109^{\circ}$ are attributed to the $P_{1}$ (white lines) and the $P_{2}$ (black lines) domains since both polar axes lie within the rotation plane and they span $\sim 109^{\circ}$. The remaining anomaly (grey line) should correspond to both the $\mathrm{P}_{3}$ and $\mathrm{P}_{4}$ domains, when the field is rotated in the high-symmetry (110) plane. The field direction [001] is even more special as the polar axes span the same $55^{\circ}$ with the field in all domains, thus, the lines should intersect each other. Such an intersection occurs at $\sim 1.5 \mathrm{~T}$ where the small deviations likely caused by a slight misorientation of the sample. However, in the same angular range phase boundaries are split around $0.75 \mathrm{~T}$ indicated by dotted lines in Fig. 3a. We found that anomalies observed on the different domains collapse into a common phase diagram when plotted on the $H_{\|}-H_{\perp}$ plane, where $H_{\|}$and $H_{\perp}$ represent the field component parallel and perpendicular to the polar axis, respectively (Fig. $3 \mathrm{~d}$ ). This confirms that the sample is in a polar multidomain state and the magnetic state stabilized by the field depends only on the angle between the polar axis and the field. We note that the finite magnetocurrent signal detected in all phases implies that the modulated magnetic states couple to the electric polarization as observed in the sister compounds $\mathrm{GaV}_{4} \mathrm{~S}_{8}$ and $\mathrm{GaV}_{4} \mathrm{Se}_{8}{ }^{38,41,44}$. This magnetoelectric coupling is allowed by the polar symmetry of $\mathrm{GaMo}_{4} \mathrm{~S}_{8}{ }^{41}$, and the magnetic field-induced changes of the spin texture modify the polarization texture.

In order to elucidate the nature of the various phases, we explored their properties for certain directions of the applied field in more details. We studied the magnetic field-induced changes in spin textures at $10 \mathrm{~K}$ by SANS experiments performed for fields parallel to the [111] axis on the (111) scattering plane. A representative set of SANS images are displayed in Fig. 4a. The smeared wavy ring of intensity present in low magnetic fields, see Fig. 2a, continuously evolves to well-defined Bragg spots in moderate fields pointing to a field-driven enhancement of the 

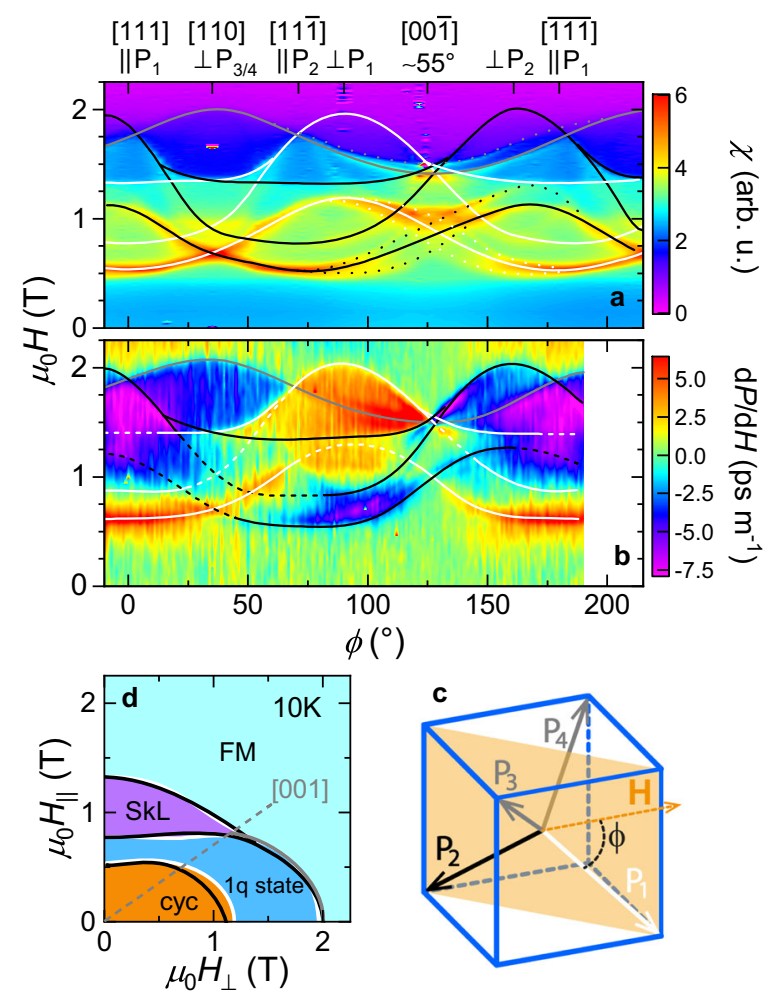

Fig. 3 Angular dependence of the magnetic phase diagram at $10 \mathrm{~K}$. Angular dependence of the magnetic susceptibility and magnetocurrent measured at $10 \mathrm{~K}$ are displayed in panel $\mathbf{a}$ and $\mathbf{b}$, respectively. Panel $\mathbf{c}$ illustrates the orientations of the four polar axes $\left(\mathrm{P}_{1-4}\right)$ and the plane where the magnetic field was rotated by angle $\phi$. The lines represent the magnetic phase boundaries for the different domains (white: $P_{1}$, black: $P_{2}$, grey: $P_{3}$ and $P_{4}$ ). Dotted lines in panel (a) represent a splitting of the phase boundary, while dashed lines in panel (b) indicate regions where the anomalies could not unambiguously be identified. The magnetic phase diagram constructed based on the angular dependence of these anomalies at $10 \mathrm{~K}$ is shown in panel d. cyc, SkL and $1 \mathrm{q}$ state respectively label the cycloidal, skyrmion lattice and a modulated phase described by a single q-vector. $H_{\|}$and $H_{\perp}$ are field components parallel and perpendicular to the polar axis, respectively.

magnetic correlation length. To make our analysis more quantitative the field dependence of the scattering intensity and the average length of the $q$-vectors are plotted in Fig. $4 c, d$, respectively, in comparison with the susceptibility shown in Fig. $4 \mathrm{~b}$. The grey and red curves are obtained by fitting a Gaussian peak on the azimuthally averaged scattering intensity around the $\langle 1 \overline{1} 0\rangle$ and $\langle 11 \overline{2}\rangle$-type directions highlighted by grey and red sectors in Fig. 4a.

The phase transitions are marked by clear anomalies of the scattering parameters. As we have demonstrated above, only the $P_{1}$ domain, responsible for the red ring in Figs. $1 d$ and 2, scatters neutrons into the red sections whereas all domains contribute to the intensity detected in the grey regions. Remarkably, our measurements evidence that modulated magnetic structures are extremely robust against a magnetic field, i.e., they extend up to 1.3 and $1.8 \mathrm{~T}$ for fields parallel to and inclined at $71^{\circ}$ from the polar axis, respectively. Such exceptional stability of modulated bulk phases has been observed so far only in centrosymmetric materials, hosting nearly atomic-scale skyrmions due to exchange frustration ${ }^{45,46}$. As the modulated phases are expected to be stable up to a critical field of order $H_{F M} \propto D^{2} / J$, this finding corroborates that the DMI is the strongest in $\mathrm{GaMo}_{4} \mathrm{~S}_{8}$ among the lacunar spinels known to host SkLs, likely due to enhancement of
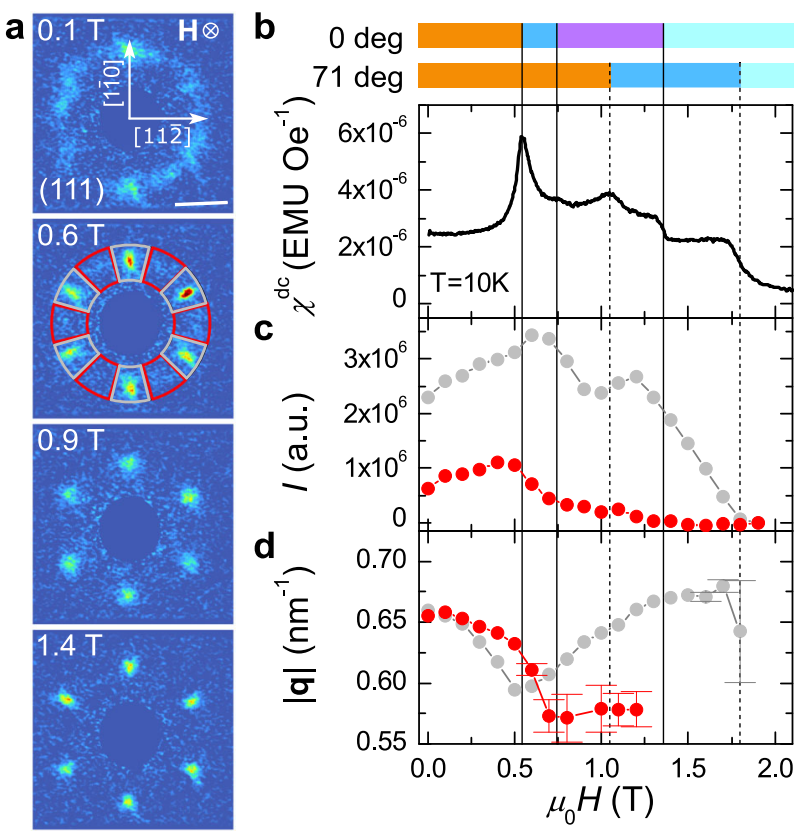

Fig. 4 Magnetic field dependence of the spin texture at $10 \mathrm{~K}$. SANS images acquired at $10 \mathrm{~K}$ in increasing magnetic fields are shown in panel a. The scale bar represents $0.5 \mathrm{~nm}^{-1}$. Both the neutron beam and the direction of the magnetic field was parallel to the [111] direction. The magnetic field dependence of the susceptibility shown in panel $\mathbf{b}$ is compared with the scattering intensity (panel c) and the position of the intensity peak (panel d) measured in the grey and red sections of the SANS patterns. The coloured stripes (same colour coding as in Fig. 3) at the top of panel (b) represent the sequence of phase transitions in $\mathrm{P}_{1}$ and $\mathrm{P}_{2-4}$ domains with polar axes spanning $0^{\circ}$ and $\sim 71^{\circ}$ with respect to the field.

the SOC from $3 d$ to $4 d$ electrons. Interestingly, after a low-field decrease $|\mathbf{q}|$ increases in higher fields in the grey region, which is unusual among skyrmion host spiral magnets. Since $|\mathbf{q}|$ does not change above $1.3 \mathrm{~T}$, where only the $\mathrm{P}_{2-4}$ domains contribute to the SANS intensity, the anomalous field induced shortening of the magnetic periodicity occurs in the $\mathrm{P}_{1}$ domain, i.e., for field parallel to the polar axis.

Since the DMI pattern dictated by the $C_{3 v}$ symmetry induces cycloidal modulation ${ }^{9}$ we assign the zero field ground state of $\mathrm{GaMo}_{4} \mathrm{~S}_{8}$ to a single-q cycloidal state (orange region in Fig. $3 \mathrm{~d}$ ) in analogy with $\mathrm{GaV}_{4} \mathrm{~S}_{8}$ and $\mathrm{GaV}_{4} \mathrm{Se}_{8}$. Although it is well established both theoretically and experimentally that the cycloidal phase is the zero field state in polar magnets of $C_{n v}$ symmetry, future polarized neutron diffraction experiments may be performed to directly prove this. The hexagonal SANS pattern with minimal intensity in the red sections, that is observed above $\sim 0.75 \mathrm{~T}$, imply the formation of a SkL state or can alternatively manifest the coexistence of cycloidal domains with $q$-vectors pointing to the different $\langle 1 \overline{1} 0\rangle$-type directions. Following the correspondence with the phase diagram of the sister compounds we rather attributed the purple phase pocket in Fig. $3 \mathrm{~d}$ to the $\mathrm{SkL}$ state. The robustness of this phase against strong tilting of the magnetic field from the polar axes implies an easy-axis type magnetic anisotropy, thus, it has the same character as in $\mathrm{GaV}_{4} \mathrm{~S}_{8}{ }^{42,47}$, which is in agreement with the predictions of recent first-principles calculations $^{48,49}$.

At finite fields between the cycloidal and the field-polarized FM states for fields perpendicular to the polar axis we detected an additional phase (coloured in blue in Fig. 3d) that is not present in the former material-specific model calculations ${ }^{42,48-51}$. We labelled it as $1 \mathrm{q}$ state since it is a modulated phase according to SANS, 
however, we cannot unambiguously determine its internal spin structure. As it possesses a finite magnetization it is likely to be a fan or a conical phase, which is not present in any other lacunar spinels. The stability range of this intermediate state narrows as the field is rotated toward the polar axis, however, it can separate the cycloidal and SkL phases even in parallel fields. The anomalous increase in $|\mathbf{q}|$ above $0.5 \mathrm{~T}$ also corresponds to the emergence of this single-q state. Although in the sister compounds the emergence of magnetic states at the polar domain walls (DWs) has been reported ${ }^{44,52}$, we rather assigned the $1 \mathrm{q}$ state to a bulk phase. At the onset of the 1q state, we found robust anomalies in all experiments including SANS. Since SANS does not possess the sensitivity to detect DW-confined states with small volume fraction, as found in $\mathrm{GaV}_{4} \mathrm{Se}_{8}{ }^{44}$, we believe the $1 \mathrm{q}$ state corresponds to a new bulk phase.

The temperature-field phase diagram is mapped by susceptibility measurements below $T_{C}=19 \mathrm{~K}$ as shown in Fig. $5 \mathrm{a}$, b for fields along [111] and [001] directions, respectively. (Field sweeps measured at constant temperatures are presented in Supplementary Fig. 1.) Schematic phase diagrams deduced for a polar monodomain sample are presented in Fig. $5 c$ and $d$ for fields respectively spanning zero and $55^{\circ}$ with the polar axis. The latter case, $\mathbf{H} \|[001]$ likely provides a representative phase diagram for the whole angular range $55^{\circ}-90^{\circ}$ as evidenced for $10 \mathrm{~K}$ in Fig. $3 \mathrm{~d}$. All phases observed at $10 \mathrm{~K}$ including the SkL state extend down



Fig. 5 Magnetic field and temperature dependence of the phase diagram for field parallel to the [111] and [001] directions. The magnetic suscpetibility as a function of magnetic field and temperature is shown for field parallel to the [111] and [001] directions in panel $\mathbf{a}$ and $\mathbf{b}$, respectively. The direction of the magnetic field with respect to the four polar axes $\left(\mathrm{P}_{1-4}\right)$ is indicated above the colour plots. The lines indicate anomalies of the susceptibility corresponding to phase transitions. For $\mathbf{H} \|[111]$ transitions occurring in the $P_{1}$ domain are highlighted by white lines whereas phase boundaries of the $\mathrm{P}_{2-4}$ domains are drawn in black. For $\mathbf{H} \|[001]$ all four domains are indistinguishable. Panel $\mathbf{c}$ and d display schematic phase diagrams deduced for a polar monodomain sample for fields respectively spanning zero and $55^{\circ}$ with the polar axis. to the lowest temperatures. In case of strong easy-axis anisotropy, modulated phases are stabilized only by thermal fluctuations as shown for $\mathrm{GaV}_{4} \mathrm{~S}_{8}{ }^{36}$, thus, the presence of cycloidal and SkL phases at low temperatures implies weaker easy-axis anisotropy in $\mathrm{GaMo}_{4} \mathrm{~S}_{8}$. The single-q state enters between the cycloidal and the SkL phases only below $13 \mathrm{~K}$ for fields applied along the polar axis, and its field stability range grows toward low temperatures. Moreover, below $6 \mathrm{~K}$ additional anomalies of the susceptibility appear suggesting the emergence of a new phase not present at $10 \mathrm{~K}$ in Fig. 3.

\section{DISCUSSION}

The main features of the phase diagram and their assignment are consistent with recent theoretical studies of $\mathrm{GaMo}_{4} \mathrm{~S}_{8}$ that combines DFT calculations and Monte-Carlo simulations ${ }^{48-50}$. These works predict only two modulated structures, the cycloidal and the Néel-type SkL states for field parallel to the polar axis. With a moderate strength of uniaxial anisotropy, these states were found to be stable down to the lowest temperatures. Although the absolute values of the interaction strength largely vary between the different calculations all of them predict a critical temperature consistent with the experiments. The ratio of the relevant DMI and the exchange interactions determining the periodicity of the cycloidal order is similar in refs. ${ }^{48,50}$, and the calculated periodicity (about $\lambda \sim 10 \mathrm{~nm}$ ) is close to the experimentally observed one. According to Nikolaev and Solovyev ${ }^{48}$, the small periodicity of the cycloidal and SkL phases is not only the consequence of the enhanced DMl due to stronger SOC, but the isotropic exchange term is also reduced from $\mathrm{GaV}_{4} \mathrm{~S}_{8}$ to $\mathrm{GaMo}_{4} \mathrm{~S}_{8}$ due to weaker screening. However, further theoretical efforts are required to understand the emergence of the 1q state between the cycloidal and the SkL phases as well as the additional low-temperature phases. An important direction of future research is, in particular, the theoretical investigation of a micromagnetic model that is able to account both for the waving of the wavevector captured by Eq. (1) as well as the magnetic phase diagram observed experimentally.

In conclusion, we studied the magnetically ordered phases of a $4 d$ cluster magnet $\mathrm{GaMo}_{4} \mathrm{~S}_{8}$ by SANS, magnetization and magneto-current measurements. We found modulated magnetic states with sub- $10 \mathrm{~nm}$ periodicity that can be attributed to the stronger DMI due to the enhanced SOC of $\mathrm{GaMo}_{4} \mathrm{~S}_{8}$, with respect to typical $3 d$ transition metal-based skyrmion hosts. The $q$-space distribution of the modulation vectors is markedly deformed, which is explained in terms of higher-order anisotropies becoming important in this $4 d$ compound. In finite fields, a series of phase transitions are observed, which is assigned to the transformation of the cycloidal state to the SkL and a new single-q state. Moreover, these modulated spin textures are coupled to the ferroelectric polarization as evidenced by our magneto-current measurements. The exceptional stability of the modulated states against magnetic fields also indicates the importance of $\mathrm{SOC}$ in $\mathrm{GaMo}_{4} \mathrm{~S}_{8}$. Our findings imply that a remarkable scaling down of the skyrmion size in bulk noncentrosymmetric materials can be achieved by exploring the plethora of $4 d$ and $5 d$ magnets.

\section{METHODS}

\section{Synthesis}

Single crystals of $\mathrm{GaMo}_{4} \mathrm{~S}_{8}$ (typical size $0.5-1 \mathrm{~mm}$ ) were grown by the flux method ${ }^{53}$. A mixture of $\mathrm{Mo}, \mathrm{MoS}_{2}$ and $\mathrm{Ga}_{2} \mathrm{~S}_{3}$ with the molar ratio of 11:3:4 was pressed into a pellet, loaded in an alumina crucible, sealed in a molybdenum tube by arc melting under argon atmosphere, and heated quickly up to $1873 \mathrm{~K}$ and cooled slowly to $1273 \mathrm{~K}$. 


\section{Small-angle neutron scattering}

SANS experiments were performed at the Oak-Ridge National Laboratory High-Flux Isotope Reactor, using the General-Purpose Small-Angle Neutron Scattering Diffractometer ${ }^{54,55}$. A single crystal with a mass of $m=112 \mathrm{mg}$ was mounted onto a rotatable sample stick with its $[1 \overline{1} 0]$ cubic direction parallel to the rotation axis. A neutron wavelength of $\lambda_{n}=6 \AA$ with $\Delta \lambda_{n}$ / $\lambda_{n}=0.13$ broadening was used with the detector set to a distance of $5 \mathrm{~m}$ from the sample, employing a collimator of the same length.

\section{Magnetization and polarization measurements}

The magnetization measurements were performed using a Quantum Design MPMS. The longitudinal magnetic susceptibility calculated from static magnetization measurements as well as the magnetocurrent were measured at $10 \mathrm{~K}$ while the field was rotated in fine steps within the $(1 \overline{1} 0)$ plane between successive field sweeps. The step size of the rotation was 1 and 2.5 degrees in the case of the magnetocurrent and the magnetization measurements, respectively. Magnetocurrent $(j=\mathrm{d} P / \mathrm{d} t)$ measurements were carried out using a Keysight Electrometer. The sample was contacted on parallel (111) faces, and correspondingly, the magnetic field-induced changes in the electric polarization component parallel to the [111] direction was detected. The magnetoelectric susceptibility was calculated from the magnetocurrent by division through the constant magnetic field sweep rate of $1.2 \mathrm{~T} / \mathrm{min}$. The field dependence of the magnetization was measured in decreasing temperature steps.

\section{DATA AVAILABILITY}

Data associated with figures are provided with this paper. The data and the data evaluation code used in the tomographic analysis of the zero-field SANS data is publicly available at GitHub: https://github.com/Buadam/SANS_Reciprocal_Space_Tomography. All other data that support the plots within this paper are available from the corresponding authors upon reasonable request.

Received: 22 October 2021; Accepted: 27 January 2022;

Published online: 07 March 2022

\section{REFERENCES}

1. Hasan, M. Z. \& Kane, C. L. Colloquium: Topological insulators. Rev. Mod. Phys. 82, 3045-3067 (2010).

2. Qi, X.-L. \& Zhang, S.-C. Topological insulators and superconductors. Rev. Mod. Phys. 83, 1057-1110 (2011).

3. Liu, Z. K. et al. Discovery of a three-dimensional topological Dirac semimetal, $\mathrm{Na}_{3} \mathrm{Bi}$. Science 343, 864-867 (2014).

4. Xu, S.-Y. et al. Discovery of a Weyl fermion semimetal and topological Fermi arcs. Science 349, 613-617 (2015).

5. Rokhinson, L. P., Liu, X. \& Furdyna, J. K. The fractional a.c. Josephson effect in a semiconductor-superconductor nanowire as a signature of Majorana particles. Nat. Phys. 8, 795-799 (2012).

6. Mourik, V. et al. Signatures of Majorana fermions in hybrid superconductorsemiconductor nanowire devices. Science 336, 1003-1007 (2012).

7. Dzyaloshinskii, I. Theory of helicoidal structures in antiferromagnets. Sov. Phys. JETP 19, 960-971 (1964).

8. Bak, P. \& Jensen, M. Theory of helical magnetic-structures and phasetransitions in MnSi and FeGe. J. Phys. C 13, L881-L885 (1980).

9. Bogdanov, A. \& Yablonskii, D. Thermodynamically stable "vortices" in magnetically ordered crystals. the mixed state of magnets. Sov. Phys. JETP 68, 101-103 (1989).

10. Nagaosa, N., Yu, X. \& Tokura, Y. Gauge fields in real and momentum spaces in magnets: monopoles and skyrmions. Philos. Trans. R. Soc. A 370, 5806-5819 (2012).

11. Neubauer, A. et al. Topological Hall-effect in the a phase of MnSi. Phys. Rev. Lett. 102, 186602 (2009).

12. Jonietz, F. et al. Spin transfer torques in MnSi at ultralow current densities. Science 330, 1648-1651 (2010).

13. Adams, $\mathrm{T}$. et al. Long-wavelength helimagnetic order and skyrmion lattice phase in $\mathrm{Cu}_{2} \mathrm{OSeO}_{3}$. Phys. Rev. Lett. 108, 237204 (2012).

14. Seki, S., Yu, X., Ishiwata, S. \& Tokura, Y. Observation of skyrmions in a multiferroic material. Science 336, 198-201 (2012).

15. Seki, S., Ishiwata, S. \& Tokura, Y. Magnetoelectric nature of skyrmions in a chiral magnetic insulator $\mathrm{Cu}_{2} \mathrm{OSeO}_{3}$. Phys. Rev. B 86, 060403 (2012).
16. White, J. S. et al. Electric-field-induced skyrmion distortion and giant lattice rotation in the magnetoelectric insulator $\mathrm{Cu}_{2} \mathrm{OSeO}_{3}$. Phys. Rev. Lett. 113, 107203 (2014).

17. Onose, Y., Okamura, Y., Seki, S., Ishiwata, S. \& Tokura, Y. Observation of magnetic excitations of skyrmion crystal in a helimagnetic insulator $\mathrm{Cu}_{2} \mathrm{OSeO}_{3}$. Phys. Rev. Lett. 109, 037603 (2012).

18. Seki, S. et al. Magnetochiral nonreciprocity of volume spin wave propagation in chiral-lattice ferromagnets. Phys. Rev. B 93, 235131 (2016).

19. Garst, M., Waizner, J. \& Grundler, D. Collective spin excitations of helices and magnetic skyrmions: review and perspectives of magnonics in noncentrosymmetric magnets. J. Phys. D: Appl. Phys. 50, 293002 (2017).

20. Zhang, X. et al. Skyrmion-skyrmion and skyrmion-edge repulsions in skyrmionbased racetrack memory. Sci. Rep. 5, 7643 (2015).

21. Parkin, S. \& Yang, S.-H. Memory on the racetrack. Nat. Nanotechnol. 10, 195-198 (2015).

22. Hanneken, $C$. et al. Electrical detection of magnetic skyrmions by tunnelling noncollinear magnetoresistance. Nat. Nanotechnol. 10, 1039-1042 (2015).

23. Maccariello, D. et al. Electrical detection of single magnetic skyrmions in metallic multilayers at room temperature. Nat. Nanotechnol. 13, 233-237 (2018).

24. Mühlbauer, S. et al. Skyrmion lattice in a chiral magnet. Science 323, 915-919 (2009).

25. Bauer, A. \& Pfleiderer, C. In Topological Structures in Ferroic Materials, (Seidel, J. ed.) 1-28 (Springer, 2016). https://doi.org/10.1007/978-3-319-25301-5.

26. Adams, T., Garst, M., Bauer, A., Georgii, R. \& Pfleiderer, C. Response of the skyrmion lattice in mnsi to cubic magnetocrystalline anisotropies. Phys. Rev. Lett. 121, 187205 (2018).

27. Fujishiro, Y. et al. Topological transitions among skyrmion- and hedgehog-lattice states in cubic chiral magnets. Nat. Commun. 10, 1059 (2019).

28. Qian, F. et al. New magnetic phase of the chiral skyrmion material Cu2OSeO3. Sci. Adv. 4, eaat7323 (2018).

29. Chacon, A. et al. Observation of two independent skyrmion phases in a chiral magnetic material. Nat. Phys. 14, 936-941 (2018).

30. Rastogi, A. \& Wohlfarth, E. Magnetic field-induced transitions in the $\mathrm{Mo}_{4}$ cluster compounds $\mathrm{GaMo}_{4} \mathrm{~S}_{8}$ and $\mathrm{GaMo}_{4} \mathrm{Se}_{8}$ showing heavy fermion behaviour. Phys. Status Solidi (b) 142, 569-573 (1987).

31. Powell, A. V. et al. Cation substitution in defect thiospinels: structural and magnetic properties of $\mathrm{GaV}_{4-x} \mathrm{Mo}_{x} \mathrm{~S}_{8}(0 \leq x \leq 4)$. Chem. Mater. 19, 5035-5044 (2007).

32. Neuber, E. et al. Architecture of nanoscale ferroelectric domains in $\mathrm{GaMo}_{4} \mathrm{~S}_{8}$. J. Phys. Condens. Matter 30, 445402 (2018).

33. Geirhos, K. et al. Orbital-order driven ferroelectricity and dipolar relaxation dynamics in multiferroic $\mathrm{GaMo}_{4} \mathrm{~S}_{8}$. Phys. Rev. B 98, 224306 (2018).

34. Geirhos, K. et al. Optical, dielectric, and magnetoelectric properties of ferroelectric and antiferroelectric lacunar spinels. Phys. Status Solidi (b) 2100160 (2021).

35. Kézsmárki, l. et al. Néel-type skyrmion lattice with confined orientation in the polar magnetic semiconductor $\mathrm{GaV}_{4} \mathrm{~S}_{8}$. Nat. Mater. 14, 1116-1122 (2015).

36. White, J. S. et al. Direct evidence for cycloidal modulations in the thermalfluctuation-stabilized spin spiral and skyrmion states of $\mathrm{GaV}_{4} \mathrm{~S}_{8}$. Phys. Rev. B 97, 020401 (2018).

37. Bordács, S. et al. Equilibrium skyrmion lattice ground state in a polar easy-plane magnet. Sci. Rep. 7, 7584 (2017).

38. Fujima, Y., Abe, N., Tokunaga, Y. \& Arima, T. Thermodynamically stable skyrmion lattice at low temperatures in a bulk crystal of lacunar spinel $\mathrm{GaV}_{4} \mathrm{Se}_{8}$. Phys. Rev. $B$ 95, 180410 (2017).

39. Pocha, R., Johrendt, D. \& Pöttgen, R. Electronic and structural instabilities in $\mathrm{GaV}_{4} \mathrm{~S}_{8}$ and $\mathrm{GaMo}_{4} \mathrm{~S}_{8}$. Chem. Mater. 12, 2882-2887 (2000).

40. Reschke, S. et al. Lattice dynamics and electronic excitations in a large family of lacunar spinels with a breathing pyrochlore lattice structure. Phys. Rev. B 101, 075118 (2020).

41. Ruff, E. et al. Multiferroicity and skyrmions carrying electric polarization in $\mathrm{GaV}_{4} \mathrm{~S}_{8}$. Sci. Adv. 1, e1500916 (2015).

42. Leonov, A. \& Kézsmárki, l. Skyrmion robustness in noncentrosymmetric magnets with axial symmetry: The role of anisotropy and tilted magnetic fields. Phys. Rev. $B$ 96, 214413 (2017)

43. Dally, R. L. et al. Magnetic phase transitions and spin density distribution in the molecular multiferroic system $\mathrm{GaV}_{4} \mathrm{~S}_{8}$. Phys. Rev. B 102, 014410 (2020).

44. Geirhos, K. et al. Macroscopic manifestation of domain-wall magnetism and magnetoelectric effect in a néel-type skyrmion host. npj Quantum Mater. 5, 1-8 (2020).

45. Kurumaji, T. et al. Skyrmion lattice with a giant topological Hall-effect in a frustrated triangular-lattice magnet. Science 365, 914-918 (2019).

46. Hirschberger, $M$. et al. Skyrmion phase and competing magnetic orders on a breathing kagomé lattice. Nat. Commun. 10, 5831 (2019).

47. Ehlers, D. et al. Exchange anisotropy in the skyrmion host $\mathrm{GaV}_{4} \mathrm{~S}_{8}$. J. Phys. Condens. Matter 29, 065803 (2016) 
48. Nikolaev, S. A. \& Solovyev, I. V. Skyrmionic order and magnetically induced polarization change in lacunar spinel compounds $\mathrm{GaV}_{4} \mathrm{~S}_{8}$ and $\mathrm{GaMo}_{4} \mathrm{~S}_{8}$ : Comparative theoretical study. Phys. Rev. B 102, 014414 (2020).

49. Kitchaev, D. A., Schueller, E. C. \& Van der Ven, A. Mapping skyrmion stability in uniaxial lacunar spinel magnets from first principles. Phys. Rev. B 101, 054409 (2020).

50. Zhang, H.-M. et al. Possible emergence of a skyrmion phase in ferroelectric $\mathrm{GaMo}_{4} \mathrm{~s}_{8}$. Phys. Rev. B 99, 214427 (2019).

51. Nikolaev, S. A. \& Solovyev, I. V. Microscopic theory of electric polarization induced by skyrmionic order in $\mathrm{GaV}_{4} \mathrm{~S}_{8}$. Phys. Rev. B 99, 100401 (2019).

52. Zuo, J. L. et al. Magnetoentropic mapping and computational modeling of cycloids and skyrmions in the lacunar spinels $\mathrm{GaV}_{4} \mathrm{~S}_{8}$ and $\mathrm{GaV}_{4} \mathrm{Se}_{8}$. Phys. Rev. Mater. 5, 054410 (2021)

53. Querré, M. et al. Electric pulse induced resistive switching in the narrow gap Mott insulator GaMo4S8. Key Eng. Mater. 617, 135-140 (2014)

54. Heller, W. T. et al. The suite of small-angle neutron scattering instruments at Oak Ridge national Laboratory. J. Appl. Crystallogr. 51, 242-248 (2018).

55. Wignall, G. D. et al. The $40 \mathrm{~m}$ general purpose small-angle neutron scattering instrument at Oak Ridge National Laboratory. J. Appl. Crystallogr. 45, 990-998 (2012).

\section{ACKNOWLEDGEMENTS}

We thank S. Picozzi, and S. Dong for useful discussions. This work was supported by the Deutsche Forschungsgemeinschaft (DFG) via the Transregional Research Collaboration TRR 80: From Electronic Correlations to Functionality (AugsburgMunich-Stuttgart) and via the DFG Priority Program SPP2137, Skyrmionics, under Grant No. KE 2370/1-1. M.G. is supported by DFG through project A07 of SFB 1143 (project-ID 247310070), DFG projects No. 270344603 and 324327023. S.B. acknowledges support by National Research, Development and Innovation Office - NKFIH, FK 153003, Bolyai $00318 / 20 / 11$ and by the BME-Nanotechnology and Materials Science FIKP grant of EMMI (BME FIKP-NAT). This research was supported by the Ministry of Innovation and Technology and the National Research, Development and Innovation Office within the Quantum Information National Laboratory of Hungary. D.S. acknowledges the FWF Austrian Science Fund Grants No. I 2816-N27 and TAI 334$\mathrm{N}$. This research used resources at the High Flux Isotope Reactor, a Department of Energy (DOE) Office of Science User Facility operated by the Oak Ridge National Laboratory.

\section{AUTHOR CONTRIBUTIONS}

T.W., Y.T. and H.N. synthesized and characterized the crystal; Á.B., K.G., L.F.K. and L.B. measured magnetization; Á.B., D.S., and L.D.-S. performed the SANS experiments; K.G. performed angular dependent magnetization and polarization measurements; Á.B. I.K. and S.B. analyzed the SANS results; M.A. and M.G. developed the theory; Á.B., I.K. M.G. and S.B. wrote the manuscript with contributions from K.G.; I.K. and S.B. planned the project.

\section{FUNDING}

Open access funding provided by Budapest University of Technology and Economics.

\section{COMPETING INTERESTS}

The authors declare no competing interests.

\section{ADDITIONAL INFORMATION}

Supplementary information The online version contains supplementary material available at https://doi.org/10.1038/s41535-022-00432-y.

Correspondence and requests for materials should be addressed to Ádám Butykai or Sándor Bordács.

Reprints and permission information is available at http://www.nature.com/ reprints

Publisher's note Springer Nature remains neutral with regard to jurisdictional claims in published maps and institutional affiliations.

(i) Open Access This article is licensed under a Creative Common Attribution 4.0 International License, which permits use, sharing, adaptation, distribution and reproduction in any medium or format, as long as you give appropriate credit to the original author(s) and the source, provide a link to the Creative Commons license, and indicate if changes were made. The images or other third party material in this article are included in the article's Creative Commons license, unless indicated otherwise in a credit line to the material. If material is not included in the article's Creative Commons license and your intended use is not permitted by statutory regulation or exceeds the permitted use, you will need to obtain permission directly from the copyright holder. To view a copy of this license, visit http://creativecommons. org/licenses/by/4.0/.

(c) The Author(s) 2022 